\title{
Electro-optical effects in 2D macroporous silicon structures with nanocoatings
}

\author{
L.A. Karachevtseva*, O.O. Lytvynenko, K.P. Konin, K.A. Parshyn, \\ O.Yu. Sapelnikova, O.J. Stronska \\ V. Lashkaryov Institute of Semiconductor Physics of NAS of Ukraine; \\ 41, prospect Nauky, 03028 Kyiv, Ukraine \\ *Corresponding author: phone +38(044) 525-23-09, e-mail: lakar@isp.kiev.ua
}

\begin{abstract}
The near-IR light absorption oscillations in 2D macroporous silicon structures with microporous silicon layers, CdTe, surface nanocrystals and $\mathrm{SiO}_{2}$ nanocoatings have been investigated. The electro-optical effect was taken into account within the strong electric field approximation. Oscillations with a giant amplitude were observed in the spectral ranges of surface level absorption. This process is because of resonance electron scattering on the surface impurity states with the difference between two resonance energies equal to the Wannier-Stark ladder due to big scattering lifetime as compared to the electron oscillation period in the strong surface electric field. The electron transitions and free electron motion are realized due to additional change in the local electric field as a result of grazing light incidence and quasi-guided mode formation.
\end{abstract}

Keywords: macroporous silicon, nanocoatings, Wannier-Stark ladder.

Manuscript received 27.05.15; revised version received 10.09.15; accepted for publication 28.10.15; published online 03.12.15.

\section{Introduction}

One of promising materials to develop 2D photonic structures is macroporous silicon that can be formed using the photoanodic etching. It is related with formation of structures with necessary geometry and high ratio between the cylindrical macropore depth and diameter [1, 2]. Presence of periodically located cylindrical pores separated by silicon columns provides large effective surface of the samples. This determines optical and photo-physical characteristics of macroporous silicon structures [3-6]. For wavelengths below the optical period of structures, the reduction of light absorption is observed owing to the guided and radiation optical modes formed by macroporous silicon as a short waveguide [6]. The absolute absorption maxima are determined by the guided optical mode position. The results obtained were explained by specificity of a macroporous silicon surface [3]. The existence of an intrinsic electric field $F_{S}=(5 \ldots 9) \cdot 10^{5} \mathrm{~V} / \mathrm{cm}$ is confirmed by electrorefectance study of macroporous silicon surfaces [7]. In view of the potential barrier on a macropore surface, one should take into account recharging of the local surface centers at energies below that of the indirect interband transition. The near-IR optical absorption in 2D photonic macroporous silicon structures was investigated with allowance made for the linear electro-optical effect [8]. The spectral dependence of optical absorption of macroporous silicon structures in the near-IR (impurity absorption) has oscillating structure and varies under the " $3 / 2$ " law at long wavelengths. It correlates with the frequency dependence of the imaginary part of permittivity for optical transitions between impurity levels and the allowed bands of a crystal in an electric field (the impurity Franz-Keldysh effect). The experimental absorption spectra of macroporous silicon agree well with the corresponding spectral dependences of the electro-optical energy and the imaginary part of permittivity in the weak electric field approximation, thus confirming realization of the impurity FranzKeldysh effect. The electric field of the reflected 
electromagnetic wave at the grazing angle of light incidence onto macropore surface changes effectively a local electric field on the macropore surface. In this paper, the near-IR light absorption oscillations of 2D macroporous silicon structures with microporous silicon layers, CdTe surface nanocrystals and $\mathrm{SiO}_{2}$ nanocoatings are investigated taking into account the electro-optical effect within the strong electric field approximation. An analysis of the experimental absorption spectra is carried out within the model of the resonant electron scattering on impurity states in strong electric field, with the difference between two resonant energies equal to the Wannier-Stark ladder. An additional electric field intensity growth due to the quasi-guided mode formation in silicon matrix with $\mathrm{SiO}_{2}$ nanocoatings was considered. A comparison of results obtained for $\mathrm{SiO}_{2}$ nanocoatings and nanocrystal surface was made.

\section{Methodology}

The samples to be studied were made of silicon wafers characterized by the [100] orientation and $n$-type of conductivity (the electron concentration $n_{0}=10^{15} \mathrm{~cm}^{-3}$ ). We used the technique of electrochemical etching at illumination of the back side of a silicon substrate (thickness $H=520 \mu \mathrm{m}$ ) [3]. The square-lattice periodic structures, as well as those with arbitrary distribution of macropores, were fabricated. The initial specimens are complex micropore-macropore silicon structures consisting of 100-nm micropore layers on macropore walls. In addition, the anisotropic etching in $10 \%$ solution of $\mathrm{KOH}$ was used to remove the microporous layers from macropore walls. Macropores with the depth $h_{p}=80 \ldots 100 \mu \mathrm{m}$, diameter $D_{p}=2 . .5 \mu \mathrm{m}$ and period $a_{p}=4 \ldots 7 \mu \mathrm{m}$ were formed.

CdTe nanocrystals of $20 \mathrm{~nm}$ in size were grown on the modified installation of metal dispersion using "a hot wall" molecular epitaxy on macroporous silicon substrates [9]. The undoped CdTe sputtered at the substrate temperature $475 \mathrm{~K}$ and source temperature $650 \mathrm{~K}$ served as substance for evaporation. The thickness of the deposited films $(200 \mathrm{~nm})$ was set by time of structure stay above the source of evaporation [10]. The layers of oxide (thickness within $70 \ldots 200 \mathrm{~nm}$ ) have been formed on macroporous silicon samples in the dry oxygen for $40(60) \mathrm{min}$ at the temperature close to $1050{ }^{\circ} \mathrm{C}\left(1200{ }^{\circ} \mathrm{C}\right)$. The 800 -nm oxide layer was formed for $50 \mathrm{~min}$ at the temperature $1100{ }^{\circ} \mathrm{C}$ in wet oxygen using a steam generator with deionized water. The thickness of oxide layer was measured using ellipsometry.

We performed optical investigations within the spectral range $1.3 \ldots 25 \mu \mathrm{m}$ using the IR Fourier spectrometer "Perkin Elmer" Spectrum BXII. The optical absorption spectra were measured at normal incidence of IR radiation on a sample (along the cylindrical macropores). The experiments were carried out in air at room temperature. The error of spectral measurements did not exceed $2 \mathrm{~cm}^{-1}$.

\section{Experimental}

Microporous silicon layers and CdTe nanocrystals. For the macroporous silicon structures with microporous layers and surface nanocrystals, light absorption increases, and an oscillating structure occurs (Fig. 1). The absorption spectra of macroporous silicon structures with $\mathrm{CdTe}$ surface nanocrystals (Fig. 1a, curve 2) and without nanocoatings (Fig. 1a, curve 3 ) have similar shapes.

The amplitude of oscillations is maximal in the spectral ranges of surface levels (organic species, $\mathrm{Si}-\mathrm{H}$, $\mathrm{C}-\mathrm{H}$ and $\mathrm{O}-\mathrm{H}$ bonds [11-14]) in the absorption spectra of macroporous silicon structures with CdTe surface nanocrystals. And $\mathrm{Si}-\mathrm{O}-\mathrm{Si}, \mathrm{Si}-\mathrm{Si}, \mathrm{Si}-\mathrm{H}_{2}, \mathrm{Si}-\mathrm{O}, \mathrm{SiCH}_{3}$, $\mathrm{C}=\mathrm{O}$ bonds were observed only in absorption spectra of macroporous silicon structures with the microporous silicon layer (Fig. 1a, curve 1) or without nanocoatings (Fig. 1a, curve 3). The form of oscillations (Fig. 1b) indicates their resonant character.

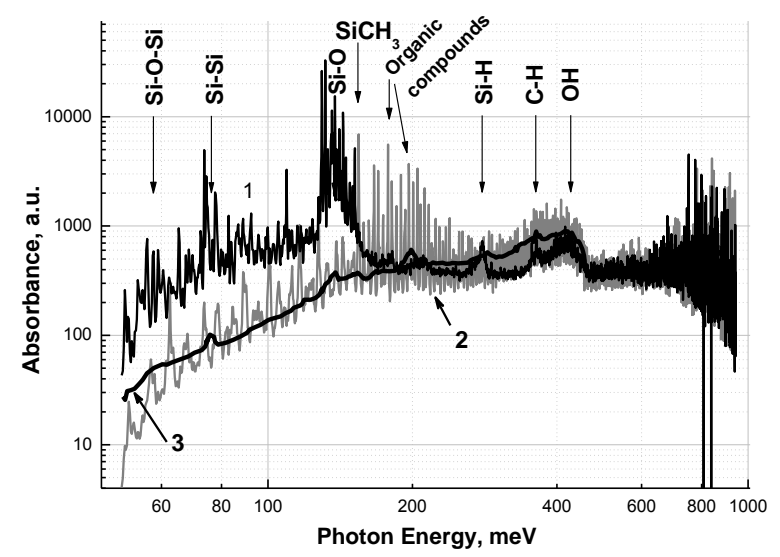

Fig. 1a. Absorption spectra of macroporous silicon structures with microporous layers (1), CdTe surface nanocrystals (2) and without coatings (3).

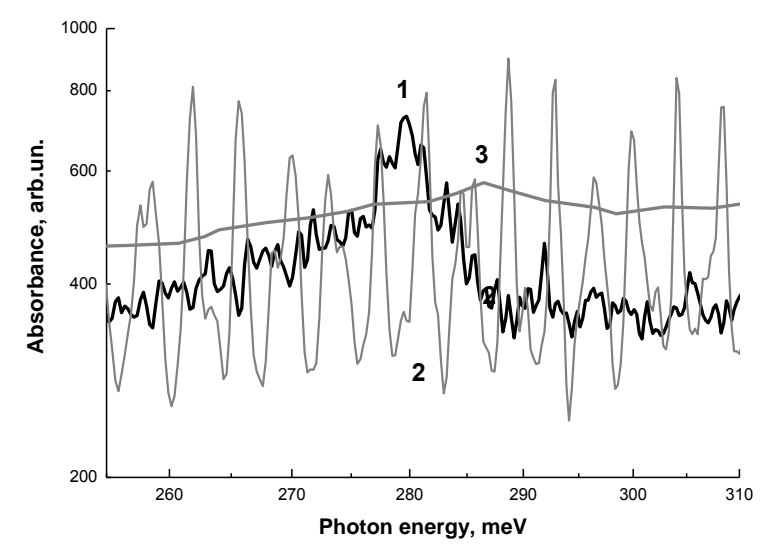

Fig. 1b. Fragments of absorption spectra of macroporous silicon structures with microporous layers (1), CdTe surface nanocrystals (2) and without coatings (3) in the vicinity of $\mathrm{Si}-\mathrm{H}$ bonds. 
The spectral positions of oscillation maxima inherent to macroporous silicon structures with surface nanocrystals and microporous layers vs oscillation number curves (Fig. 2a, curves 1,2) are straight lines, and the oscillation period is almost constant (Fig. 2b). The oscillation energies $\Delta E$ lie between 0.7 to $2 \mathrm{meV}$ for microporous layers and lie between 0.7 to 2 and 3 to $5 \mathrm{meV}$ for CdTe surface nanocrystals. The oscillations of small amplitude and other spectral position of oscillation maxima (Fig. 2a, curve 3) have been investigated for macroporous silicon structures without nanocoatings [8]. The experimental absorption spectra of macroporous silicon agree well with the corresponding spectral dependences of the electro-optical energy and the imaginary part of permittivity in the weak electric field approximation, thus confirming realization of the impurity Franz-Keldysh effect.

$\mathrm{SiO}_{2}$ nanocoatings. For macroporous silicon structures with $\mathrm{SiO}_{2}$ nanocoatings, light absorption increases and oscillating structure occurs, too (Fig. 3a, curves 1 and 2). We observed the essential absorption growth in the spectral range of $\mathrm{Si}-\mathrm{O}, \mathrm{Si}-\mathrm{H}, \mathrm{O}-\mathrm{H}$ bonds and organic compounds. The amplitude of oscillations is maximal within the spectral ranges of surface level absorption (Fig. 3b).

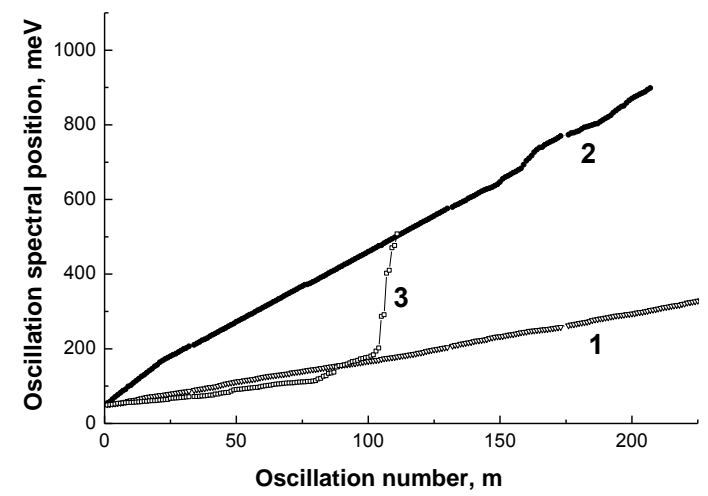

Fig. 2a. The spectral position of oscillation maxima of macroporous silicon structures with microporous layers (1), CdTe nanocrystals (2) and without coatings (3) [7] as a function of oscillation number.

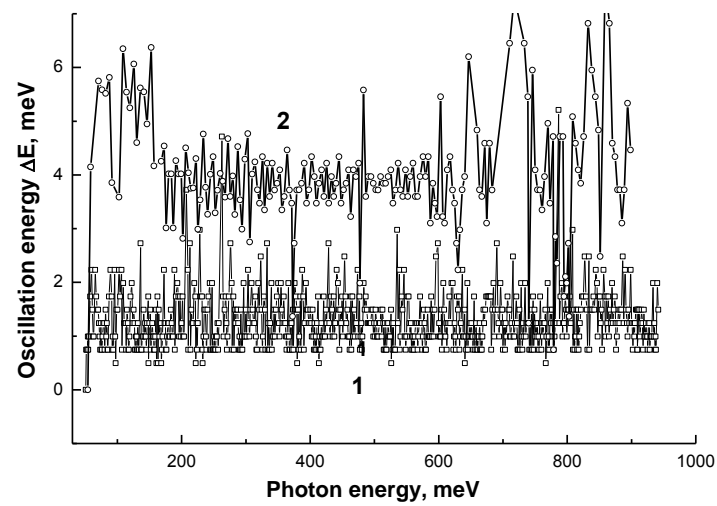

Fig. 2b. Spectral dependences of the oscillation period of macroporous silicon structures with microporous layers (1), CdTe (2).

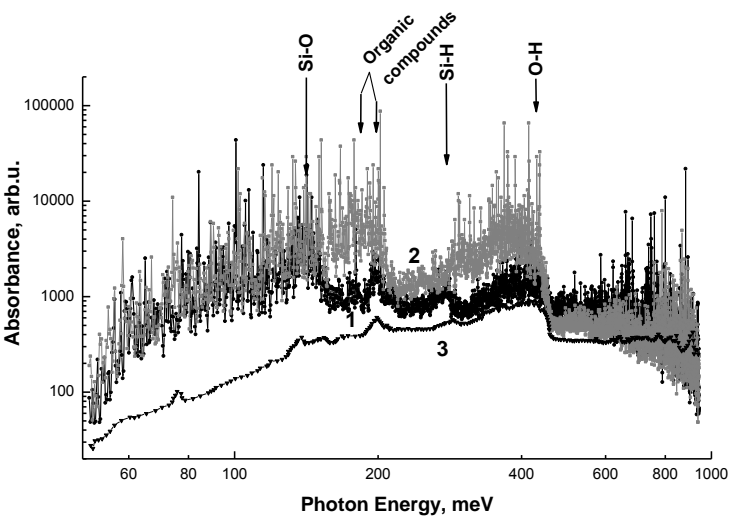

Fig. 3a. Absorption spectra of macroporous silicon structures with $\mathrm{SiO}_{2}$ nanocoating $70 \mathrm{~nm}(1)$ and $800 \mathrm{~nm}$ (2) thick and without coating (3).

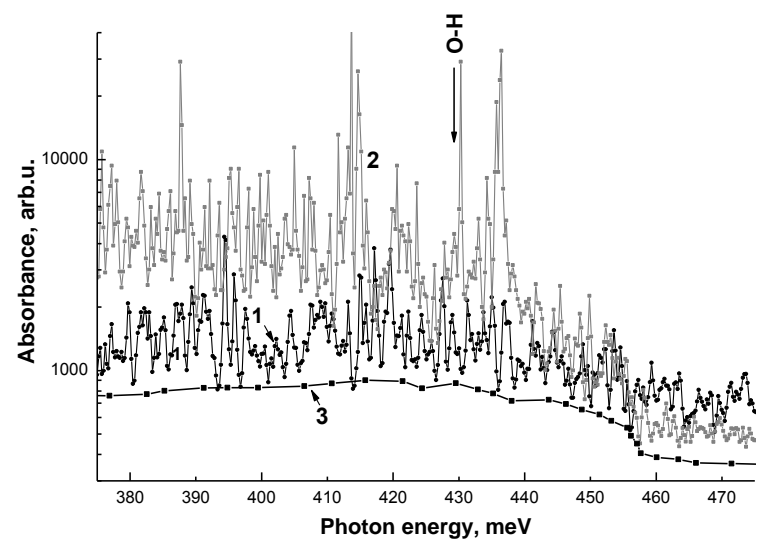

Fig. 3b. Fragment of absorption spectra of macroporous silicon structures with $\mathrm{SiO}_{2}$ nanocoatings $70 \mathrm{~nm}$ (1) and $800 \mathrm{~nm} \mathrm{(2)}$ thick and without coating (3).

The spectral positions of oscillation maxima in the macroporous silicon structures with $\mathrm{SiO}_{2}$ nanocoatings and without coating are rather different (Fig. 4a). The dependence of oscillation maxima of macroporous silicon structures with $\mathrm{SiO}_{2}$ nanocoating on the oscillation number has peculiarities at energies of 0.25 , 0.4 and $0.7 \mathrm{eV}$. In addition, the oscillation period fluctuates about a constant value at low spectral energies and becomes quadratic in the photon energy depending on the $\mathrm{SiO}_{2}$ nanocoating thickness (Fig. 4b).

\section{Discussion}

We observed the oscillating structure in the absorption spectra of macroporous silicon structures with surface nanocrystals. The amplitude of oscillations is maximal in spectral ranges of organic species, $\mathrm{Si}-\mathrm{H}, \mathrm{C}-\mathrm{H}$ and $\mathrm{O}-\mathrm{H}$ bonds. The obtained results indicate strong effect of impurity states on the surface of macroporous silicon structures with nanocoatings. It may result from scattering of both electromagnetic radiation and electrons on the impurity states. The form of oscillations (Fig. 1b, 3b) indicates resonant character of scattering. 


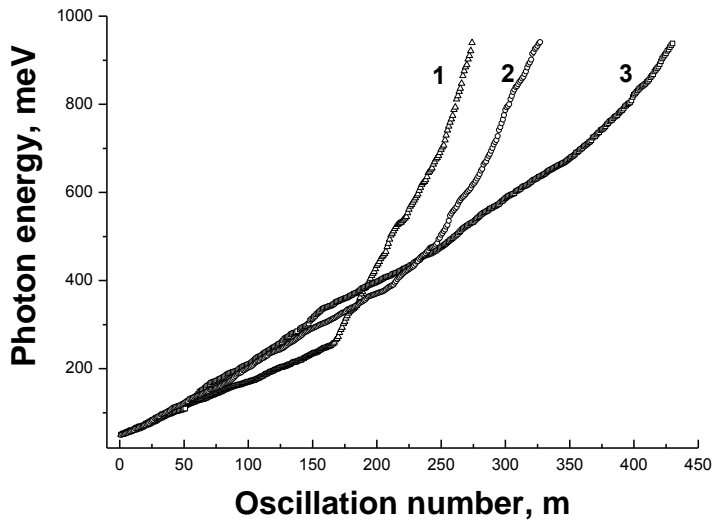

Fig. 4a. The spectral position of oscillation maxima in the macroporous silicon structures with $\mathrm{SiO}_{2}$ nanocoatings of $70 \mathrm{~nm}(1), 200 \mathrm{~nm}(2)$ and $800 \mathrm{~nm}(3)$ thickness.

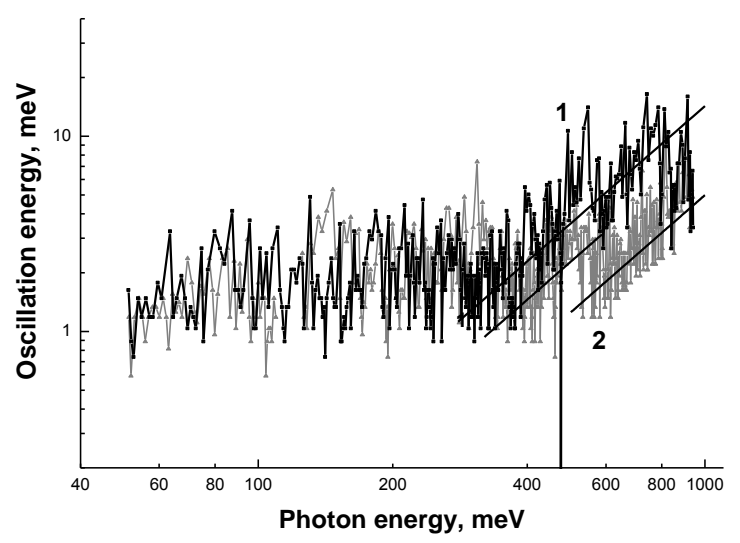

Fig. 4b. Spectral dependences of the oscillation energy in the macroporous silicon structures with $\mathrm{SiO}_{2}$ nanocoatings of $200 \mathrm{~nm}(1)$ and $800 \mathrm{~nm}(2)$ thickness.

The oscillations of small amplitude in macroporous silicon structures without nanocoatings correspond to the weak electric field approximation [8]. The macroporous silicon structures with surface nanocrystals investigated in this work have higher surface potential of nanocoatings with surface bonds. Therefore, the onset of oscillations with a giant amplitude can be attributed to the electro-optical processes in strong electric fields. Moreover, the constant oscillation period (Fig. 2b) may specify the realization of the Wannier-Stark effect on randomly distributed surface bonds in nanocrystals. The method for experimental observation of Wannier-Stark ladder was proposed by Berezhkovskii and Ovchinnikov [15]. It was shown that the scattering amplitude has resonant behavior in the case of electron scattering by impurities. If the electric field is directed along the $x$-axis of the crystal, then electron scattering occurs in the plane $(y, z)$, and the difference between two resonant energies is approximately equal to WannierStark ladder. In our case, an electric field of "siliconnanocoating" heterojunctions on the macropore surface is directed perpendicularly to the surface, too (Fig. 3), and surface states that scatter electrons are concentrated perpendicularly to the $x$-direction in the plane $(y, z)$ that is the plane of resonant scattering.

Let us consider a semiconductor with the dispersion law $E(k)=E_{0}-\Delta\left(\cos k_{y} a+\cos k_{z} a\right)$, where $k$ is a quasimomentum with components $k_{y}, k_{z}, E_{0}$ - energy corresponding to the midgap, $\Delta$ - energy equal to $1 / 6$ of the band gap, $a$ - lattice parameter. The wave function in the Wannier representation was written as [15]:

$$
\left\langle j \mid \psi_{E}\right\rangle=\left\langle j \mid \Phi_{E}\right\rangle+\frac{\left\langle j\left|\hat{G}_{0}(E)\right| 0\right\rangle V_{0}\left\langle 0 \mid \Phi_{E}\right\rangle}{1-V_{0}\left\langle 0\left|\hat{G}_{0}(E)\right| 0\right\rangle}
$$

Here, the first (second) term describes the incident wave (scattered waves); $j$ numbers the lattice site, $\hat{G}_{0}(E)$ is the Green operator, $V_{0}$ - impurity potential. The complex energies for which the denominator of the second term becomes zero correspond to the resonances in electron scattering

$1 / V_{0}=\left\langle 0\left|\hat{G}_{0}(E)\right| 0\right\rangle$

at $E=\varepsilon-i \Gamma(\Gamma>0)$. The difference of two neighbouring resonance energies is approximately equal to the value of the step in the Wannier-Stark ladder.

The fact is that the levels of the Wannier-Stark ladder have a certain width $\Gamma$, while its detection requires that this width should be less than the difference of energies of adjacent levels, $\Gamma<F d$. The contributions to the width $\Gamma$ come from the interband interaction, electron-phonon interaction, and interaction with impurity atoms. The interband [16] and the electronphonon [17] interactions have been studied, and such interactions do not break the Wannier-Stark ladder. The influence of impurities on the Wannier-Stark ladder and calculation of the width of the Wannier-Stark ladder levels $\Delta E$ due to scattering from impurities were considered by Berezhkovskii and Ovchinnikov [18]. The Wannier-Stark ladder is not broken by impurities, if the intervals between transitions due to scattering by impurity atoms with the lifetime $\tau$ are bigger than the period of electron oscillations in external field, $T_{\mathrm{B}}\left(\tau / T_{\mathrm{B}}>1\right)$, where $T_{\mathrm{B}}=2 \pi h / \Delta E, \tau$ is equal to $1 / W(W$ is the probability for an electron to leave the state per unit time due to scattering by an impurity atom at lattice site). The following estimate of the probability $W$ for electron to leave the state per unit time due to scattering by an impurity atom at lattice site was obtained: $W<2 V_{0} N_{i} /\left(N_{h}\right)$, where $V_{0}$ is the impurity potential, $N_{i}$ impurity concentration and $N \approx\left(a^{2}\right)^{-1}$ - density of states. As a result, the inequality $\tau / T_{\mathrm{B}}>1$ passes to $N_{i}<\Delta E /\left(4 \pi a^{2} V_{0}\right)$. Using the latter inequality, we found a numerical estimate for the impurity concentration.

We obtained the surface impurity concentration in macroporous silicon structures by using the method of the photoconductivity dependence on the distance between macropores [19] and the temperature dependences of the photocarrier lifetime in macroporous 
silicon [20]. From the experimental temperature dependences of the photocarrier lifetime in 2D macroporous silicon structures, the dimensionless surface potential $y_{0}$ is about 12 at room temperature, which corresponds to the equilibrium surface band bending of about $0.31 \mathrm{eV}$ and to the surface impurity concentration $N_{i}=5 \cdot 10^{10} \mathrm{~cm}^{-2}$ for the electron concentration $n_{0}=10^{15} \mathrm{~cm}^{-3}$ of the investigated macroporous silicon samples. In this case, the WannierStark ladder is preserved within the whole spectral range for macroporous silicon structures with microporous layers and surface nanocrystals (Fig. 6). The Bloch oscillation time is equal to $T_{\mathrm{B}} \approx(4 \ldots 8) \cdot 10^{-12} \mathrm{~s}$ for macroporous silicon structures with surface nanocrystals, and $T_{\mathrm{B}} \approx(1 \ldots 4) \cdot 10^{-11} \mathrm{~s}$ for macroporous silicon structures with microporous layers. The lifetime ratio is $\tau / T_{\mathrm{B}}>1$ within the whole spectral region studied for macroporous silicon structures with surface nanocrystals. And the inequality $\tau / T_{\mathrm{B}}>1$ for this lifetime ratio is satisfied within the whole investigated spectral region for macroporous silicon structures with $\mathrm{SiO}_{2}$ nanocoatings (Fig. 7), if taking into account that the surface impurity concentration for macroporous silicon structures, $N_{i}$, is less than $10^{11} \mathrm{~cm}^{-2}$.

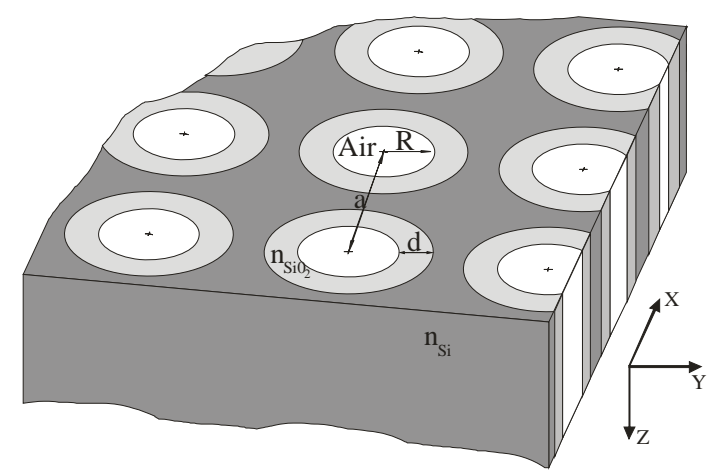

Fig. 5. Fragment of system considered.

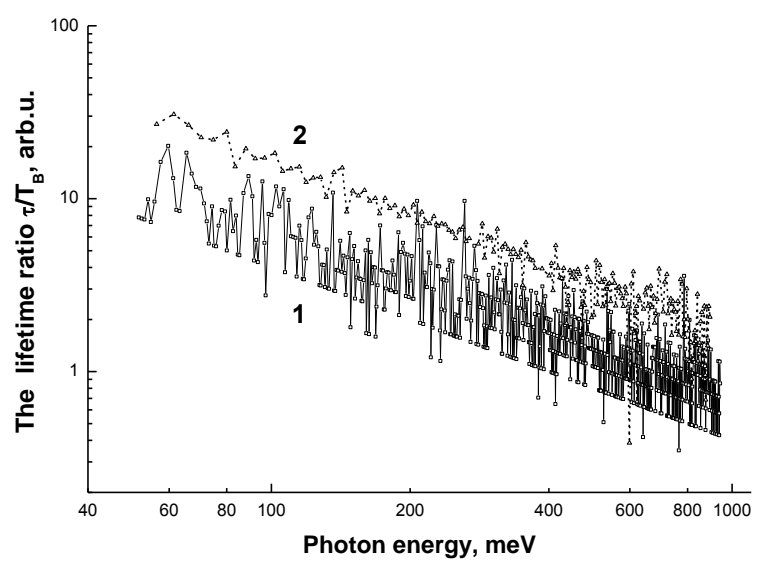

Fig. 6. Spectral dependence of the lifetime ratio $\tau / T_{\mathrm{B}}$ for macroporous silicon structures with microporous layers $(l)$ and CdTe (2) surface nanocrystals.

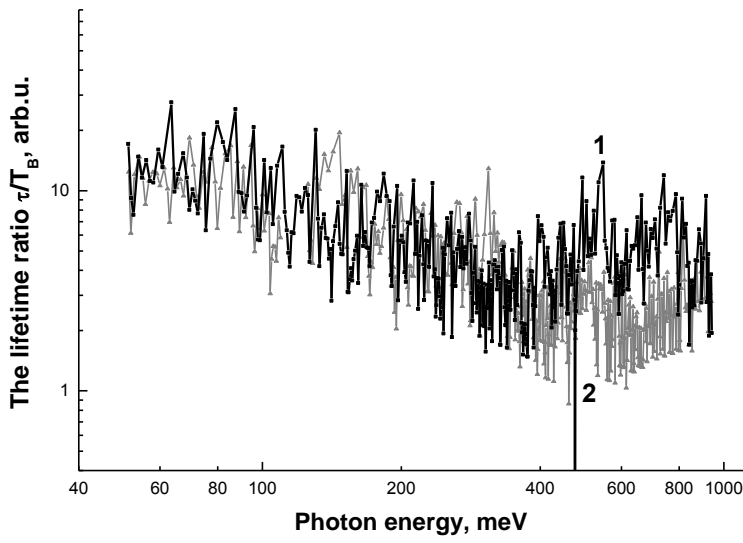

Fig. 7. Spectral dependence of the lifetime ratio $\tau / T_{\mathrm{B}}$ for macroporous silicon structures with $\mathrm{SiO}_{2}$ nanocoatings of $200 \mathrm{~nm}(1)$ and $800 \mathrm{~nm}(2)$ thickness.

The big scattering time is needed to make possible generation of well-separated oscillations. The oscillation periods $\Delta E$ for macroporous silicon structures with surface nanocrystals and microporous layers depend mainly on the band gap of the nanocrystal material (see Fig. $2 b$ and Table). The electric field intensity $F$ is equal to $10^{4} \ldots 10^{5} \mathrm{~V} / \mathrm{cm}$ for $F=\Delta E / a$, and $(3 \ldots 8) \cdot 10^{3} \mathrm{~V} / \mathrm{cm}$ for $F=\Delta E / d$ (see Table). The latter value of electric field intensity is too small, the Bloch time is bigger than the relaxation time, and therefore neither Bloch oscillations nor Wannier-Stark ladders have been observed yet. This proves the validity of the model of electron oscillations in the atomic lattice.

Usually, the basic sources of external electric field at semiconductor surface are the charge of electron levels and built-in charge in semiconductor surface oxide [7]. The oscillation period and electric field intensity of macroporous silicon structures with $\mathrm{SiO}_{2}$ nanocoatings fluctuate around constant value at low spectral light energies and become quadratic in the photon energy depending on geometrical sizes of silicon matrix and $\mathrm{SiO}_{2}$ nanocoatings (see Fig. 8). The electric field intensity growth corresponds to the quasi-guided mode formation [21] in the silicon matrix (minimal distance between macropores) with $2 \rho_{\mathrm{Si}}=a_{p}-\left(D_{p}+d_{\mathrm{SiO}_{2}}\right)$ and in silicon column with $2 \rho_{\mathrm{Si}}=1.4\left(a_{p}-\left(D_{p}+d_{\mathrm{SiO}_{2}}\right)\right)$. The mode parameter [22] $Q_{\mathrm{Si}} \sim k \rho_{\mathrm{Si}}$ is determined by the beginning of the photon energy quadratic growth (Fig. 8, curves 1-4).

In general, at grazing angle of light incidence [23], the electric field of the reflected electromagnetic wave changes the local electric field in the near-surface region of the macropore walls with the thickness $d \approx 0.1 \lambda$ for the wavelength $\lambda$. Let us consider that $d$ is determined by the electric component of electromagnetic wave with $\hbar \omega$ and by the change of built-in electric field $\Delta F_{s}(d=$ $\hbar \omega /\left(e \cdot \Delta F_{s}\right)$ ). Indeed, under our experimental condition of the grazing angle of light incidence onto the macropore surface, the electric field intensity on macroporous 
Table. Structure parameters and the electric field intensity.

\begin{tabular}{|c|c|c|c|c|c|c|}
\hline $\begin{array}{c}\text { Nanocrystal } \\
\text { material }\end{array}$ & $\begin{array}{c}\text { Oscillation } \\
\text { period, } \mathrm{meV}\end{array}$ & $\begin{array}{c}\text { Lattice } \\
\text { parameter, } \\
a, \mathrm{~nm}\end{array}$ & $\begin{array}{c}\text { Band } \\
\mathrm{gap}, \mathrm{eV}\end{array}$ & $\begin{array}{c}\text { Nanocrystal } \\
\text { size, } \\
d, \mathrm{~nm}\end{array}$ & $\begin{array}{c}\text { Electric field } \\
\text { intensity } \\
F=\Delta E / a, \mathrm{~V} / \mathrm{cm}\end{array}$ & $\begin{array}{c}\text { Electric field } \\
\text { intensity } \\
F=\Delta E / d, \mathrm{~V} / \mathrm{cm}\end{array}$ \\
\hline $\mathrm{Si}$ & $1.4 \pm 0.6$ & 0.54 & 1.1 & $2 \ldots 3$ & $(1.5 \ldots 4) \cdot 10^{4}$ & $(3 \ldots 9) \cdot 10^{3}$ \\
\hline $\mathrm{CdTe}$ & $3.9 \pm 0.5$ & 0.65 & 1.6 & 20 & $(6 \ldots 8) \cdot 10^{4}$ & $(2 \ldots 3) \cdot 10^{3}$ \\
\hline
\end{tabular}

silicon surface for structures with $\mathrm{SiO}_{2}$ nanocoatings is about $F_{s}+\Delta F_{s}$ with $\Delta F_{s} \approx \hbar \omega /\left(0.1 \lambda_{1}\right) \sim \hbar \omega^{2}$ according to the experiment (Fig. 4). The light wavelength is equal to $\lambda_{1}=\lambda / n_{i}\left(n_{i}\right.$ is effective refractive index of pores with $\mathrm{SiO}_{2}$ nanocoatings or refractive index of $\mathrm{SiO}_{2}$ nanocoatings).

The electric component of electromagnetic wave changes from $E=\hbar \omega$ to zero when going from the silicon matrix to macropore. It corresponds to a reverse bias at the surface barrier, and its band bending grows. This effect is strong for light energy corresponding to the spectral range of quasi-guided (leakage) mode formation in the silicon matrix (see Fig. 8). This result differs from that for macroporous silicon structures without nanocoatings [8], where the $\Delta F_{s}$ change was observed over for all the spectral range investigated due to the low surface barrier. On the other hand, the electric field intensity in the macroporous silicon structures with microporous layer and surface nanocrystals [21] does not change in the short wavelength spectral range (Fig. 8, curve 5). Constant oscillation period (and electric field intensity) in macroporous silicon structures with nanocrystals may be attributed to absence of the quasiguided mode formation in the silicon matrix due to strong light scattering by nanocrystals. As a result, the local

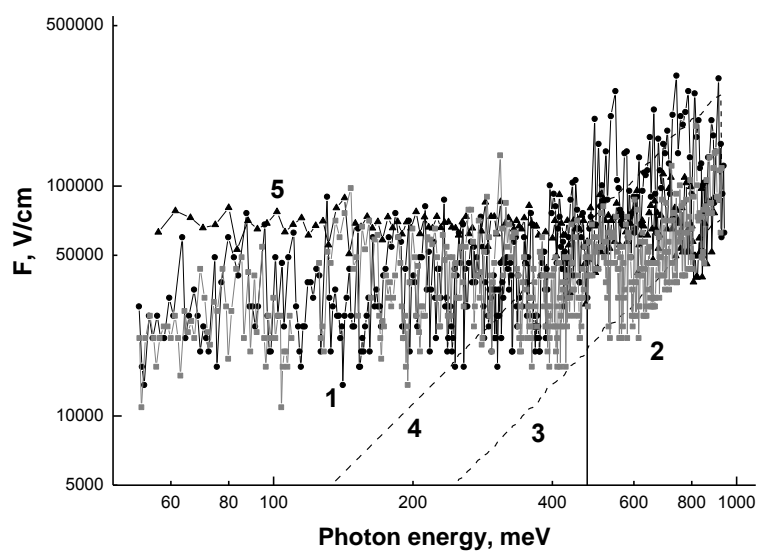

Fig. 8. Spectral dependences of the electric field intensity $F$ on macroporous silicon surface for structures with $\mathrm{SiO}_{2}$ nanocoatings of $200 \mathrm{~nm}(1)$ and $800 \mathrm{~nm}$ (2) thickness; and spectral dependences of the electric field intensity as a result of quasi-guided mode formation in the silicon matrix $(3,4)$; spectral dependences of the electric field intensity in the macroporous silicon structures with $\mathrm{CdTe}$ surface nanocrystals (5). electric field variation in the heterojunction area became negligible in comparison with macroporous silicon structures with $\mathrm{SiO}_{2}$ nanocoating. In addition, the nanocrystals form local contact with silicon surface, thus increasing the constant local electric field intensity and decreasing the surface level concentration. That is why harmonic oscillations with low period deviations were measured in absorption spectra of the macroporous silicon structures with the contacted CdTe nanocrystals (Fig. 1).

In general, the absorption spectra of macroporous silicon without nanocoatings agree well with the corresponding spectral dependences of the electrooptical energy and the imaginary part of permittivity in the weak electric field approximation, thus confirming realization of the impurity Franz-Keldysh effect [8]. At a higher light intensity, the impurity absorption bands become wider, the broadening parameter $\Gamma$ grows, and oscillations are not observed (Fig. 1a, curve 3). But the oscillator structure was restored in the absorption spectra of macroporous silicon with nanocoatings. In the latter case, recharging the local surface centers at energies below those of the indirect interband transition (the impurity Franz-Keldysh effect) was added by resonance electron scattering (the Wannier-Stark effect) due to a higher electric field intensity at the silicon-nanocoating interface and electron motion from silicon matrix to the surface states in the heterojunction area. Really, the near-IR absorption is a result of electron transitions from the $v$-band to empty acceptor surface levels, and the Fermi level shifts to the $c$-band. It increases the free electron concentration in the $c$-band. The electron transitions and free electron motion are realized due to additional change in the local electric field as a result of grazing light incidence and quasi-guided mode formation (for $\mathrm{SiO}_{2}$ nanocoatings only). Observation of coherent electronic wave-packet oscillations in a semiconductor heterostructure at room temperature was reported in [24], too.

\section{Conclusions}

We have observed well-separated oscillations in absorption spectra of macroporous silicon structures with surface nanocrystals and with $\mathrm{SiO}_{2}$ nanocoatings. The amplitude of oscillations is maximal within the spectral ranges of organic species, $\mathrm{Si}-\mathrm{H}, \mathrm{C}-\mathrm{H}$ and $\mathrm{O}-\mathrm{H}$ bonds. The results obtained indicate strong influence of impurity states on the surface of macroporous silicon 
structures with nanocoatings. It is caused by resonant electron scattering by the impurity states in the electric field of "silicon-nanocoating" heterojunction on macropore surface. The constant oscillation period specifies realization of the Wannier-Stark effect on the randomly distributed surface bonds on nanocrystals. The amplitude of scattering has resonant behavior in the case of electron scattering by impurity, and the difference between two resonant energies is equal to the WannierStark ladder. The oscillation periods $\Delta E$ of macroporous silicon structures with surface nanocrystals and microporous layers mainly depend on the band gap of the nanocrystal material. The electric field intensity $F$ is $10^{4} \ldots 10^{5} \mathrm{~V} / \mathrm{cm}$ for $F=\Delta E / a$, thus indicating validity of the model of electron oscillations in the atomic lattice.

The Wannier-Stark ladder is not broken by impurities, if the intervals between the transitions due to scattering by impurity atoms with the lifetime $\tau$ are wider than the period of electron oscillations in the external field, $T_{\mathrm{B}}$. The lifetime ratio is $\tau / T_{\mathrm{B}}>1 \mathrm{in}$ all the spectral regions considered for macroporous silicon structures with CdTe surface nanocrystals, taking into account that the surface impurity concentration for macroporous silicon structures is less than $N_{i} \approx$ $5 \cdot 10^{10} \mathrm{~cm}^{-2}$. And the inequality $\tau / T_{\mathrm{B}}>1$ holds over the whole spectral regions considered for macroporous silicon structures with $\mathrm{SiO}_{2}$ nanocoatings, taking into account that the surface impurity concentration $N_{i}$ for macroporous silicon structures is less than $5 \cdot 10^{11} \mathrm{~cm}^{-2}$.

The oscillation period and electric field intensity in the macroporous silicon structures with $\mathrm{SiO}_{2}$ nanocoatings fluctuate around constant value at low photon energies and become quadratic in photon energy depending on the geometrical sizes of silicon matrix and $\mathrm{SiO}_{2}$ nanocoatings. The relevant electric field intensity growth corresponds to quasi-guided mode formation in the silicon matrix (minimal distance between the macropores) and in the silicon column. The local electric field variation was observed in macroporous silicon structures without nanocoatings within all the investigated spectral range due to the low surface barrier. The electric field intensity in the macroporous silicon structures with microporous layer and surface CdTe nanocrystals does not change due to the high surface barrier, strong light scattering by nanocrystals and absence of quasi-guided mode formation in the silicon matrix.

In general, for macroporous silicon structures with nanocoatings, the near-IR absorption is a result of electron transitions from the $v$-band to empty acceptor surface levels, and the Fermi level shifts to the $c$-band. It increases the free electron concentration in the $c$-band. The electron transitions and free electron motion are realized due to additional change in the local electric field as a result of grazing light incidence and quasiguided mode formation (for $\mathrm{SiO}_{2}$ nanocoatings only). Resonant electron scattering gives rise to the resonances of the permittivity at room temperature and a corresponding change in absorption.

\section{References}

1. A. Birner, R. Wehrspohn, U. Gösele and K. Busch, Silicon-based photonic crystals // Adv. Mater. 13(6), p. 377-388 (2001).

2. L.A. Karachevtseva, Two-dimensional photonic crystals as perspective materials of modern nanoelectronics // Semiconductor Physics, Quantum Electronics \& Optoelectronics, 7(4), p. 430435 (2005).

3. L.A. Karachevtseva, A.E. Glushko, V.I. Ivanov et al., Out-of-plane optical transmittance of 2D photonic macroporous silicon structures // Semiconductor Physics, Quantum Electronics \& Optoelectronics, 10(2), p. 51-57 (2007).

4. A. Glushko, L. Karachevtseva, Photonic band structure in oxidized macroporous silicon // OptoElectronics Review, 14(3), p. 201-203 (2006).

5. A. Glushko, L. Karachevtseva, PBG properties of three-component 2D photonic crystals // Photonics and Nanostructures, 4(3), p. 141-145 (2006).

6. L.A. Karachevtseva, N.I. Karas', V.F. Onischenko, F.F. Sizov, Surface polaritons in 2D macroporous silicon structures // Int. J. Nanotechnology, 3(1), p. 76-88 (2006).

7. R.Yu. Holiney, L.A. Matveeva, E.F. Venger, O.A. Litvinenko, and L.A. Karachevtseva, Electroreflectance study of macroporous silicon structures // Appl. Surf. Sci. 172(3), p. 214-219 (2001).

8. L.A. Karachevtseva, V.I. Ivanov, O.O. Lytvynenko, K.A. Parshin, O.J. Stronska, The impurity FranzKeldysh effect in 2D photonic macroporous silicon structures // Appl. Surf. Sci. 255(5), p. 3328-3331 (2008).

9. W. Huber and A. Lopez-Otero, The electrical properties of CdTe films grown by hot wall epitaxy // Thin Solid Films, 58(1), p. 21-27 (1979).

10. Ye. Bilevych, A. Soshnikov, L. Darchuk et al., Influence of substrate materials on the properties of CdTe thin films grown by hot-wall epitaxy // $\mathrm{J}$. Crystal Growth, 275(1-2), p. 1177-1181 (2005).

11. A.G. Cullis, L.T. Canham, P.D.J. Calcott, The structural and luminescence properties of porous silicon // J. Appl. Phys. 82(3), p. 909-965 (1997).

12. L.T. Canham, A. Loni, P.D.J. Calcott et al., On the origin of blue luminescence arising from atmospheric impregnation of oxidized porous silicon // Thin Solid Films, 276, p. 112-115 (1996).

13. F.L. Galeener, Band limits and the vibrational spectra of tetrahedral glasses // Phys. Rev. B, 19, p. $4292-4297$ (1979).

14. R.M. Almeida, C.G. Pantano, Study of chemical composition of porous silicon surface // J. Appl. Phys. 68, p. 4225-4243 (1990).

15. A.M. Berezhkovskii, A.A. Ovchinnikov, Electron scattering on impurities in crystal in a constant electric field // Theor. \& Mat. Phys. 39(2), p. 284288 (1979). 
16. A.M. Berezhkovskii, A.A. Ovchinnikov, Real width of electron levels in crystal in a constant electric field // Fizika tverd. tela, 18, p. 3273-3278 (1976), in Russian.

17. R. Kümmel, H. Rauh, E. Bangert, Theory of StarkLadder Currents // phys. status solidi (b), 87(1), p. 99-103 (1978).

18. A.M. Berezhkovskii, A.A. Ovchinnikov, Influence of impurities on the Wannier-Stark ladder in semiconductor in a strong electric field // phys. status solidi (b), 110(2), p. 455-459 (1982).

19. V.I. Ivanov, L.A. Karachevtseva, N.I. Karas et al., Photoeffects in silicon with regular structure of pores // Semiconductor Physics, Quantum Electronics \& Optoelectronics, 10(4), p. 72-76 (2007).

20. L. Karachevtseva, V. Onyshchenko and A. Sachenko, Photocarrier transport in 2D macroporous silicon structures // Opto-Electronics Review, 18(4), p. 394-399 (2010).

21. L. Karachevtseva, S. Kuchmii, O. Lytvynenko, F. Sizov, O. Stronska and A. Stroyuk, Oscillations of light absorption in 2D macroporous silicon structures with surface nanocoatings // Appl. Surf. Sci. 257, p. 3331-3335 (2010).

22. A.W. Snyder, J.D. Love, Optical Waveguide Theory. London, New York, Charman and Hall, 1983.

23. N.J. Harrick, Optical spectrum of the semiconductor surface states from frustrated total internal reflections // Phys. Rev. 125, p. 1165-1170 (1962).

24. T. Dekorsy, R. Ott, H. Kurz, K. Köhler, Bloch oscillations at room temperature // Phys. Rev. B, 51(23), p. 17275-17278 (1995). 